\title{
Composite Technical Solutions in Combination with Modern Software Products as Multifunctional Equivalents of Complex Integrative Inventions
}

\author{
Konstantin A. Piliugin, Ph.D. \\ Voronezh, Russian Federation
}

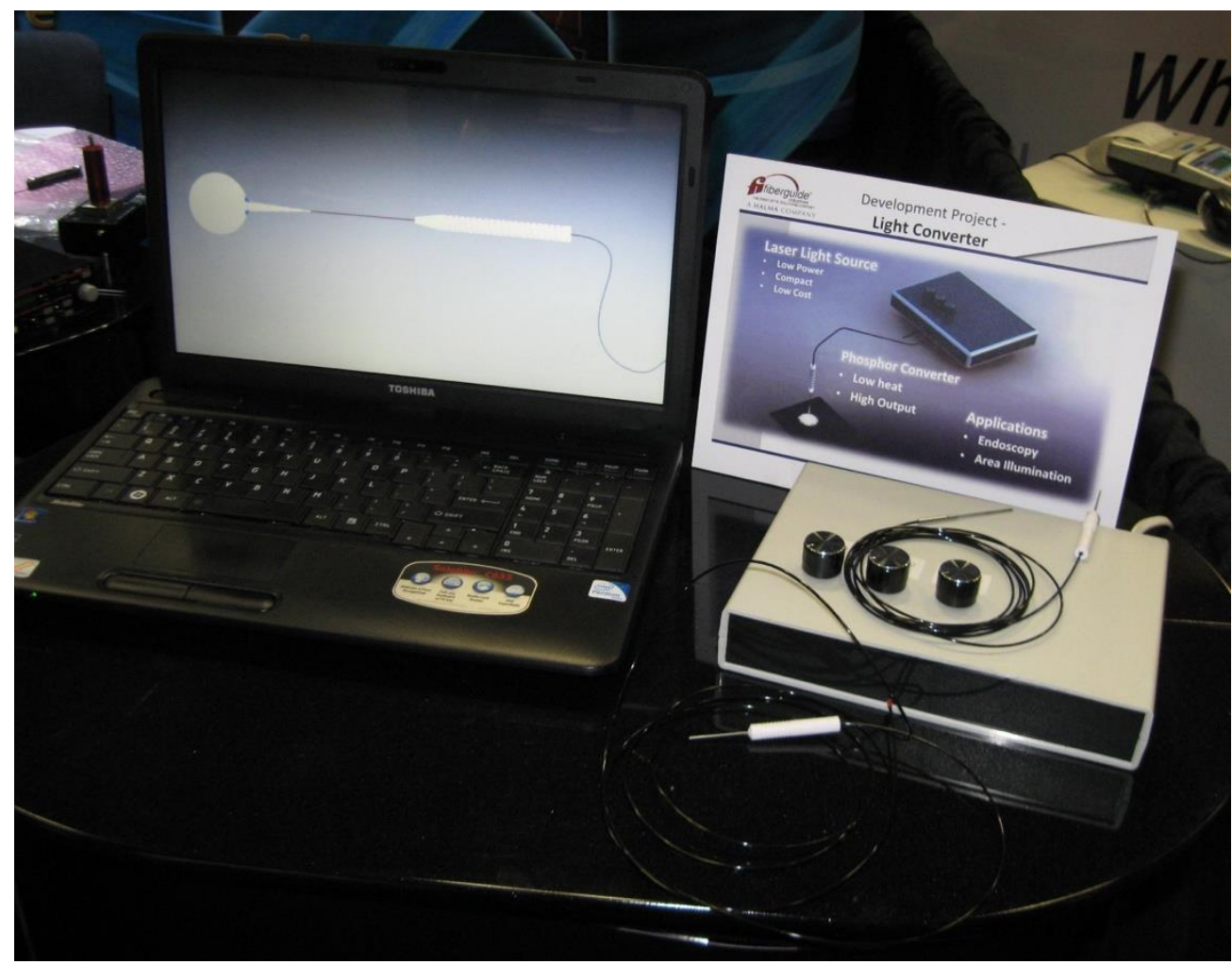

Figure 1. Example of a composite technical solution in combination with modern complex software products and base units made as multifunctional equivalents of complex integrative inventions in several related technological areas, including laser diodes, precision optical cables with composite luminophores and encoding and decoding of disposable laser tools with correction of emission spectrum using composite luminophores deposited on an optical cable.

All inventors know that sometimes technical solutions are created that, in real conditions, operate, work and solve many tasks, which, when stated, pushed the inventor to innovative 
analysis and initiated his/her purposeful creative activity, but there are at first glance interesting technical solutions, but as a result of a deeper analysis, turns out that they are created in isolation from reality and solve absolutely nothing, except for the realization of ambitious claims to at least some (most often not very useful, and sometimes useless at all) - idea in the field of engineering and technology.

In addition, technical solutions arising in a local area necessarily directly or indirectly affect existing stereotypes and even psychological barriers that arise on their basis, which prevent from overcoming common technical and specific technological contradictions that have arisen on the basis and in the course of development of these psychological barriers.

Even twenty years ago, the need for inventions of the second group and the equally important need to take into account the influence of psychological barriers were somehow justified by their supporting role as the basis for the selective choice of the most effective technical solutions.

The emergence of information technologies and a sharp reduction in the time cycle intended for the development and transformation of an inventive idea into a really necessary, demanded and marketable product, complication of the technical and technological components of new products, causing a proportional increase in the cost of manufacturing prototypes of the invented product and their testing, make us completely consider the possibility of creating technical solutions with innovative support functions in a completely new way.

Now, if the inventor wants his/her innovative ideas to be used, he/she must be more versatile and must have both the technique of foresight, intuition and, to a certain extent, developed imagination, and also be a practically universal specialist, at least feeling and (better) well aware of the commercial and consumer demands of the market regardless of the technical and technological stereotypes combined with them and arising on their basis and under their influence including psychological barriers.

There are several basic directions that have a decisive influence on the fate of new ideas in today's conditions and, taking them into account can provide real and high level of commercial success, or their neglect will forever close the way for the idea to be implemented in any commercial form. 
I propose to consider some of these basic areas (of course, the scope of the article and the monograph only allow doing it in abstract form):

\section{Availability of fundamentally new materials}

Consider, for example, two new technological areas, creation of efficient light sources based on blue laser (laser diode) radiation and creation of composite food products based on efficient mixing systems and hydrodynamic activation.

Both of the above technological areas for development require structural materials, which, due to their properties, in each direction, will provide parameters that cannot be obtained using conventional materials.

The possibility of using new materials is not absolute and their application requires a primary composite solution.

For example, lasers require a highly efficient heat sink and a system for scattering current pulses, and its implementation requires changes in the design of a laser case in order to use composite diamond-copper material which can perform these functions due to its parameters and properties.

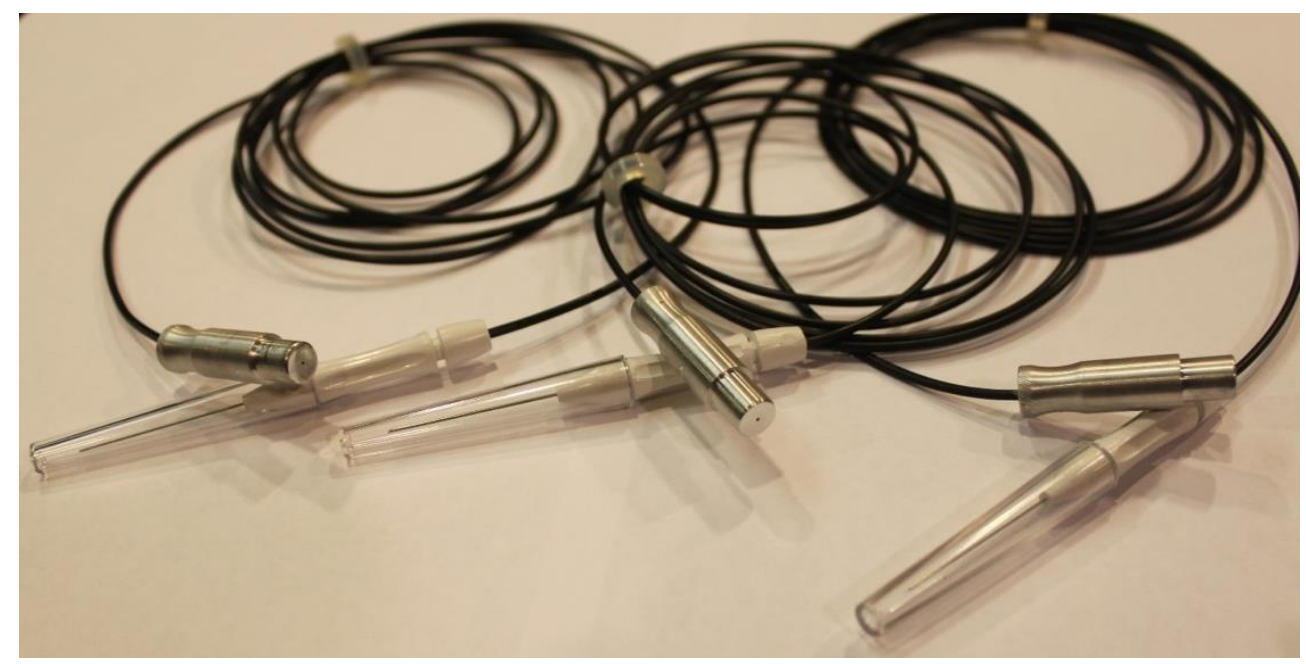

Figure 2. The figure shows disposable laser tools, which are also composite technical solutions in a lower hierarchy of complex technical solutions, where fundamentally new materials determine the possibility of achieving an ideal final result while maintaining the 
design concept without significant changes, as well as preserving the principles of directed impedance and resonance coding and decoding to ensure the identification of replaceable disposable instruments.

But it turns out that replacement of the material does not solve all the complex problems faced by the creators of new lasers; What is the solution? A solution can be a composite scheme, when, for example, in addition to the material, a radio frequency driver is introduced into the system of technical composition, which, in combination with the material that performs the functions of cooling and dissipating excess heat and current pulsations, allows the laser to provide the pumping mode, which in turn allows for a laser with a power of 1 watt, to introduce a pumping current of, say, 2 watts. 


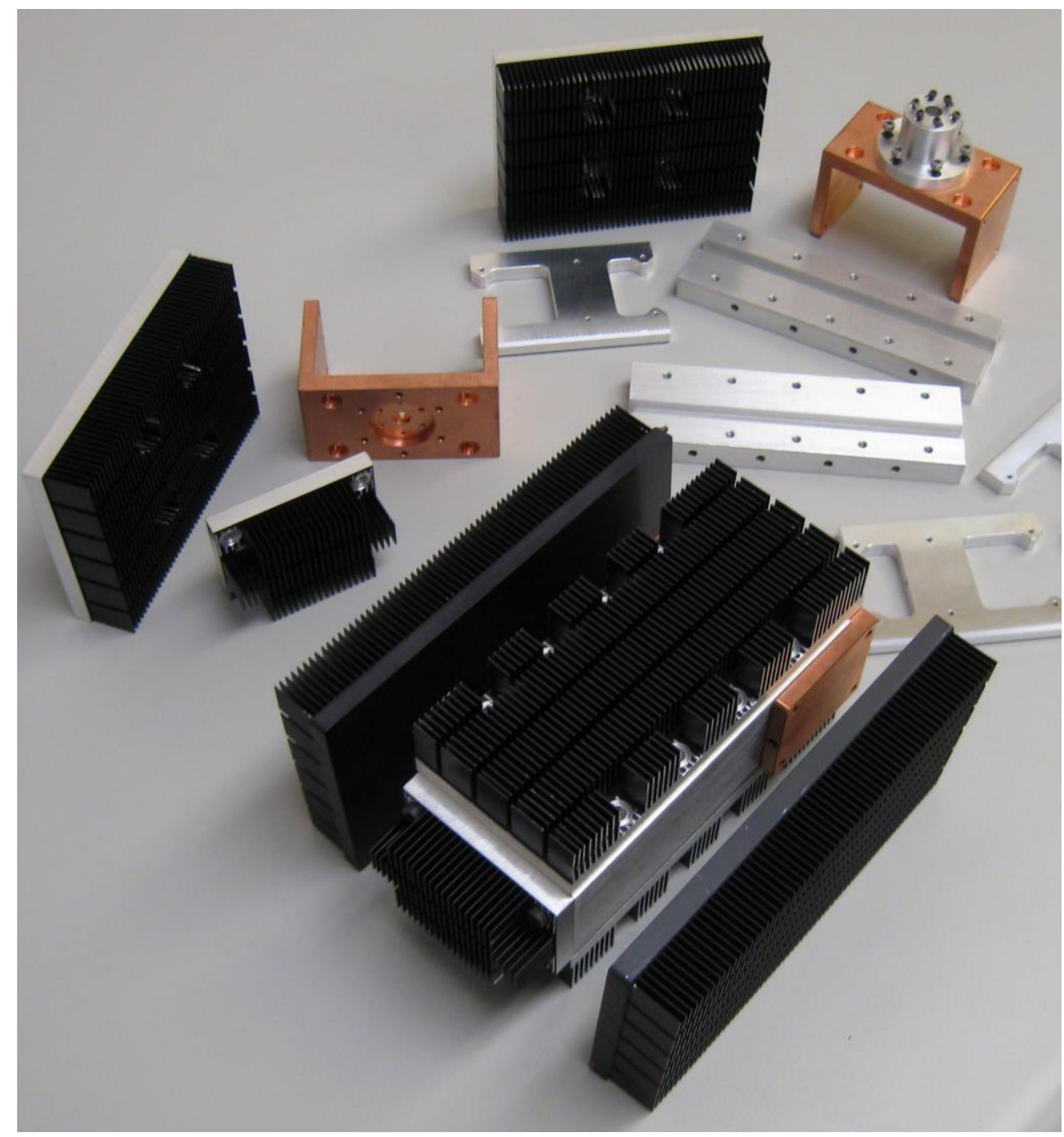

Figure 3. The figure shows the laser module components containing basically the options of diamond and copper composites, changing the basic parameters of the technical capabilities of the housing modules for laser diodes and providing, among other things, the possibility for a significant increase in the speed of the electronic components of the system, which, due to these composites, allow applying progressive integrative inventions - RHYTHM - boards, which in turn are a complex integrative invention.

This is already the so-called leap of effect and quality, and this illustrates the fact that in modern conditions the most optimal technical solution bringing the desired result is a composite technical solution. 
In the second example, the composite solution looks quite different, in it, a fundamentally new material is the final product of the technology, which can be represented as a new homogeneous food composition, produced just and only thanks to the composite system of the homogenization process in the process of mixing and churning.

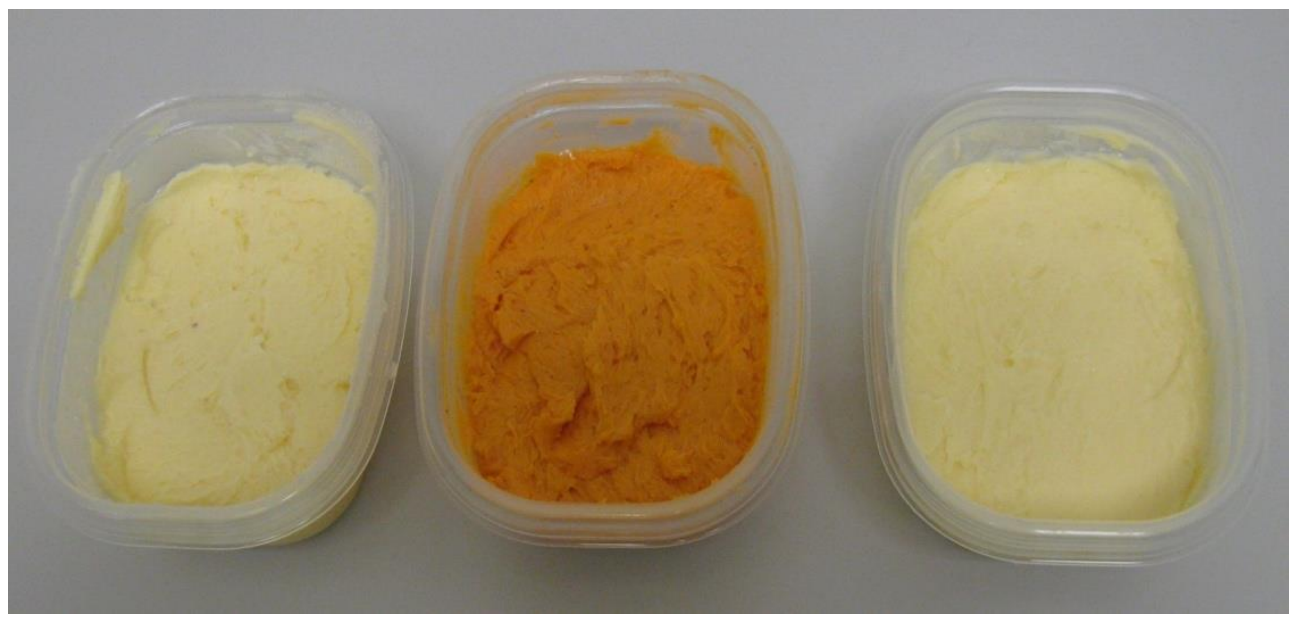

Figure 4. The figure shows samples of composite butter containing more than 30\% fortified filler; In such composite solutions, the main factor ensuring the efficiency of the technology is the factor of the technique of churning of the composite butter using an earlier integrative solution, the so-called dynamic churning in the "tumbling barrel" system.

\section{Presence of composite materials}

Information on scientific and technological developments of composites is eagerly awaited in the market and new composite materials are constantly appearing, and their presence makes adjustments to the design of new products and the process of their manufacture.

In order to link these new developments with the enormous scientific, technical and technological experience gained in research and development enterprises, we consider, as an example, the method of creating nano-composites, where the main component is a nanopowder of synthetic diamond, by the way, its production technology was born in the Ukrainian Institute of Superhard Materials. 
A significant novelty added to this technology was the method of high-speed nanocoatings on nano-diamond powders made of the most ductile metals, such as copper, silver, gold, platinum, and with further introduction of these components into a complex technological composition, with the introduction in the process of the operation of initiating the flow properties of plastic metals at low temperatures and subsequent plastic calibration under ultrahigh pressure, of thus obtained nano-capsules; Thus, we see in this composite solution a sequentially horizontally oriented integration of the technique of precision nano-coatings and the technique of initiating cold flow in a material deposited using the same coatings.

Thus, this publication aims to show that the existing level of research and development in the field of composite materials science allows, when the task is correctly stated, the developers of one component of the composition and, with in-depth cooperation with the developers of similar technologies whose product is another component of the composition, creating technology and materials relevant and popular in the market.

Now we can trace how the process of forming a composition of technologies and materials is developing.

From the creation of complex integrated, energy-rich semiconductor devices, in particular, semiconductor lasers (laser diodes), the problem of effective heat sink, heat dissipation, current pulses dispersion and fluctuations appeared.

The cause of this problem was the absence among structural materials, alloys and various combinations, and combinations of materials, of the ability to reliably and steadily perform these functions.

All materials and their derivatives in a certain degree did not suit the developers and operators, and only with the advent of the possibility of creating complex composite technical solutions, problems of this level can be solved.

\section{New areas of component manufacturing technology}

In composite technical solutions, methods and technologies for manufacturing product parts and components are the most important factor determining general introduction of this product. 
As an example, consider a nozzle or fuel injector of an internal combustion engine. This is one of the most popular products. Over a billion of such injectors is manufactured in the world per year.

For such a product, its key value is determined by several key factors, - diameter of outlet orifices and ensuring tightness at high fuel pressures (up to 2,000 atmospheres). Making holes using the conventional technology determines the limits of the minimum diameter of holes, and since at high pressures holes are required with the diameter of microns, the technology for making these holes should be, for example, laser; In this case, the inventor of a new injector should provide a composite component of the invention novelty, expressed in accordance with specific requirements of the equipment for laser drilling.

\section{New consumer standards}

Constantly changing conditions and consumer demands, in combination with local consumption standards, with cultural and national traditions underlying local consumption standards, create informal, but tacitly present conditional consumer standards.

If the goal of the inventor is to ensure commercial success for his/her invention, then a basic understanding of the current consumer standard criteria, parameters of technical, operational and functional characteristics of a new product, should be an essential part of its commercial strategy.

If technological risk is excluded because inspections and tests of a new product have yielded positive results, the risk of commercial failure for this, technologically quite successful product, really remains, if the inventors and their commercialization partners did not take into account or did not understand the essence of the consumer standard for their product.

\section{New environmental standards}

As is known, the restrictive requirements of environmental standards are becoming more stringent. While working on the invention of a new product, which application somehow affects the limits of permissible parameters regulated by environmental protection standards, it is necessary to provide for full compliance with the requirements and restrictions of existing standards when operating a new product. 
As a rule, existing standards are constantly being improved and the requirements that are in effect in the current year will be consistently made more stringent in a few years.

This is especially important for various power equipment and internal combustion engines.

There are cases when an internal combustion engine was invented with an innovative cylinder layout, with an extremely efficient fuel economy, but the concentration level of nitrogen oxides and soot in the exhaust gases exceeded the permissible standard, which should take effect two years later.

This was enough for the newest engine to be subject to revision, where its technical solution was changed to a composite level with the inclusion in it, integrated with previous technical solutions, of an innovative system of treatment and activation of fuel, which allowed to reduce the concentration of harmful substances in exhaust gases.

\section{Fashion effect}

Even for high-tech products, fashion exists, as a developed by time and practice kind of integral complex stereotype that combines both objective and subjective commercial and operational factors.

Subjectivity of this concept is not always explained by direct logic methods, but it must be taken into account by the inventor both when developing a new product and when preparing its presentation for potential partners and consumers.

Very often, an innovative product lacks elements, factors or signs that future consumers wait for and will favorably accept with interest, while the authors concentrate on purely technical aspects and offer them nothing at all that they want to hear and see.

\section{Availability and constant intensive development of software products}

Significant complication of equipment and especially various types of electronic and microelectronic devices has fundamentally changed the principles of their protection as objects of complex, multidimensional and multifunctional intellectual property.

For such objects, the essential differences regarding purely design features, circuit solutions, combinations of these solutions do not determine all aspects of the invention, since today very often all of the listed features and differences can be realized into a working system 
or prototype only under certain conditions and capabilities of manufacturing and control technology, and very often it is the manufacture that determines the basic properties of the invention.

The development of processor control systems also determines viability of the technical solution, and therefore the algorithm or algorithms, programs, feedback between the elements of the structure or scheme become or have confidently become an organic part of the technical solution underlying the claimed invention.

Thus, in one description it is necessary to combine or integrate several different technologies and this combination, the identified possible channels and links of such integration should be presented in the invention formula in such a way as to prevent the patent department expert from doubting the unity of all integrated distinctive features of a future invention and divide it into a number of local technical solutions based on one technological direction.

Possibilities of patenting in the field of information technology affect a huge layer of activity in modern society. And if very recently it was possible to characterize or limit a certain technological sector very accurately, with the advent of high technologies and their branches, information technologies, into all areas of human activity, such classification capabilities and protective mechanisms have changed significantly and transformed into a new system of technical, commercial and legal relationships.

In almost all, even relatively simple processes, their structure becomes integrative and includes technological methods, methods and systems never used before, and, moreover, the integration of classical technical solutions with new capabilities provided by information technologies radically changes the concept of invention itself.

This factor that has arisen at the junction of technologies significantly changes the attitude to formulation and protection of those elements and their combinations, which in such new conditions can be qualified as integrative technical solutions that correspond to the key signs of the invention and based on composite design and technological elements. 


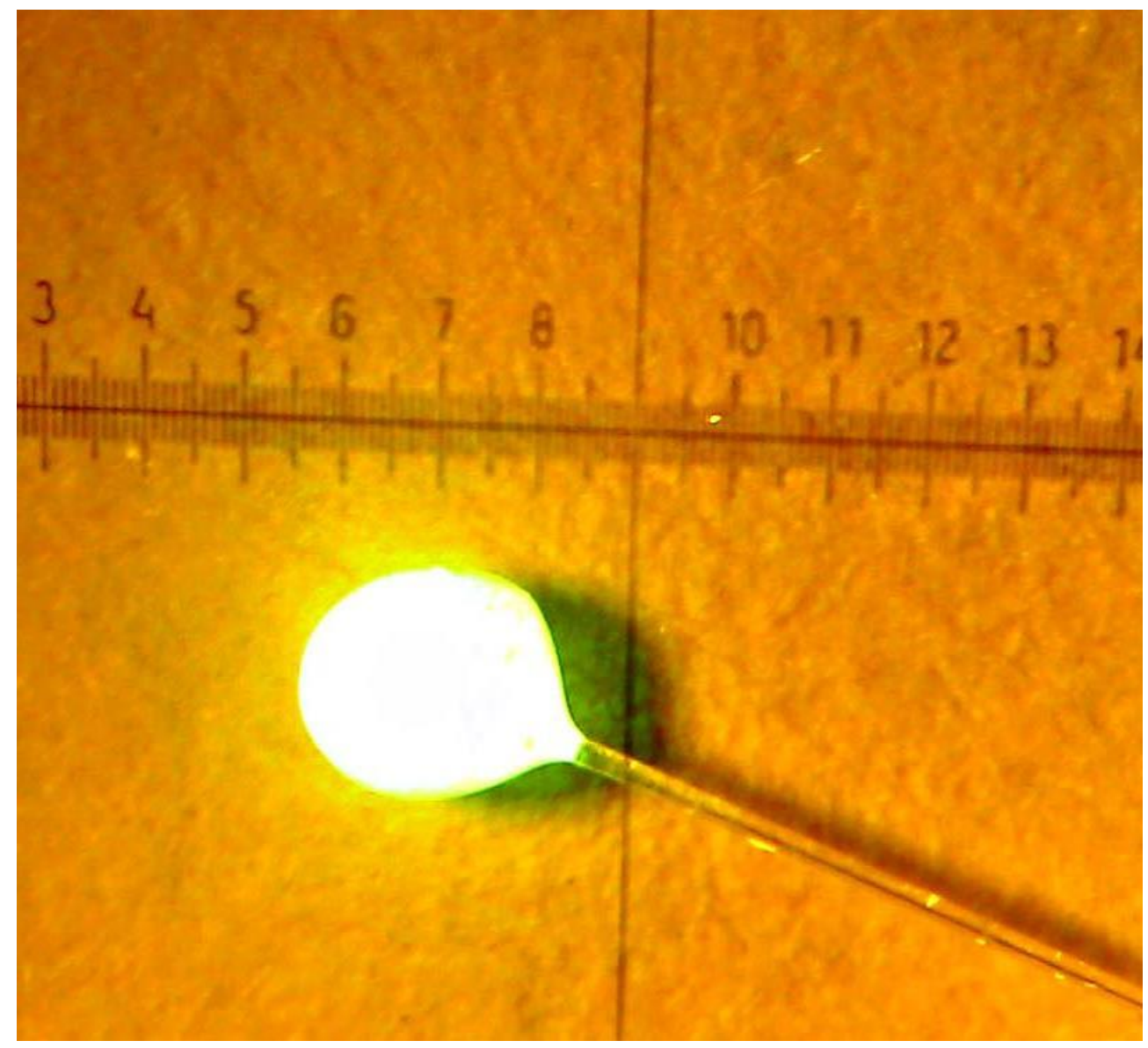

Figure 5. The figure shows a drop of luminophore at the end of an optical cable connected to a laser diode; Shooting takes place in such a way that after it is completed, the resulting amount of information provides the full amount and cycles of computer modelling and simulation of all processes to identify mistakes and directions for correcting them at the next design stages.

\section{Change of rules and criteria of industrial design}

Introduction of new technologies, the use of new materials, replacement of traditionally accepted production methods with unusual ones, which help and are an indispensable condition of a technological leap or breakthrough and increase production efficiency, are now called innovative processes. 


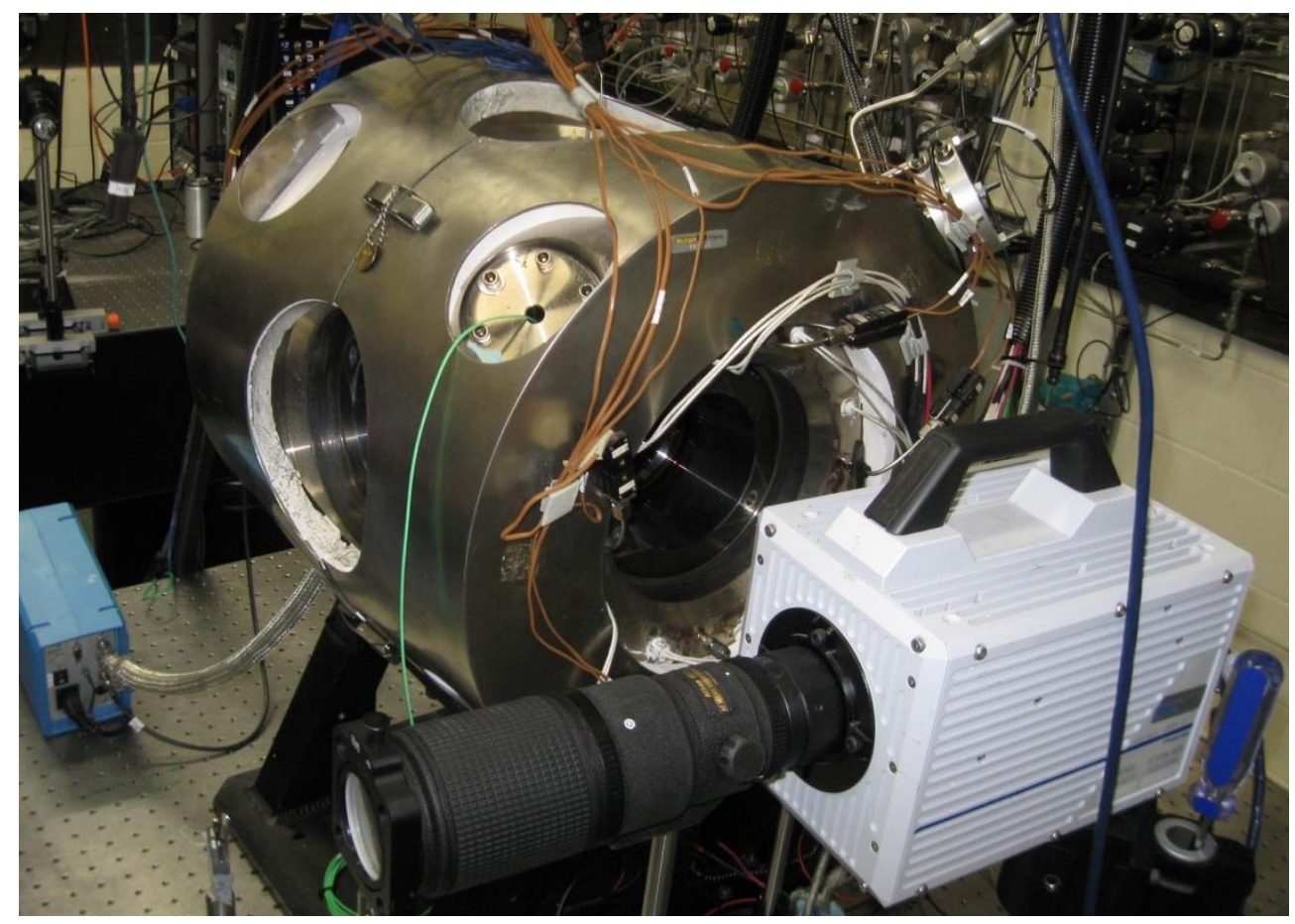

Figure 6. The figure shows the experimental design of a system for testing and verifying special single-cylinder experimental engines capable of recording the processes of injection and combustion in real time; such tests are performed before transition to full-engine testing to eliminate the risks and verify the need for additional changes in the design and mode of engine operation at a low level of costs

This process in conditions of different technical and technological cultures, in conditions of different levels of starting positions for the beginning of the initiated process of innovation, may differ significantly, but there is an urgent need to start such a process and this fact does not cause any doubts.

In recent years, the economy in almost all industrialized countries has become and continues to become of an increasingly innovative nature.

And if at the beginning of the process innovation breakthrough had a local value and was experienced in the field of high technology, microelectronics and so-called nano-technology, today the innovation process is becoming more and more focused on classic, basic technology, energy, medicine, transport, i.e. covers all basic spheres of human life. 
In order to increase competitiveness of their products and technologies, entrepreneurs are constantly forced to look for new ways to improve efficiency, reduce energy intensity and overall energy costs, increase environmental safety and economic stability within each individual enterprise or company.

New features in the design and performance testing of technical solutions also add elements of composite design solutions, and they become the main criteria for the tools and methodology of industrial design.

\section{A new look at the new product longevity}

More recently, the product longevity was one of the most important criteria determining its commercial value. Currently, with a constant decrease in the time from the start of a new product sales period to the beginning of a newer product sales period, this time period is so small that there is often no point in the innovation process to focus and spend effort and money on an excessive increase in longevity, which exceeds the period between the beginning of the exploitation of an existing product and the entry of a newer or modernized product to the market. Since this period may differ significantly for different types of product, the concept of longevity may be blurred in time and is not critical as the purpose of the invention. 


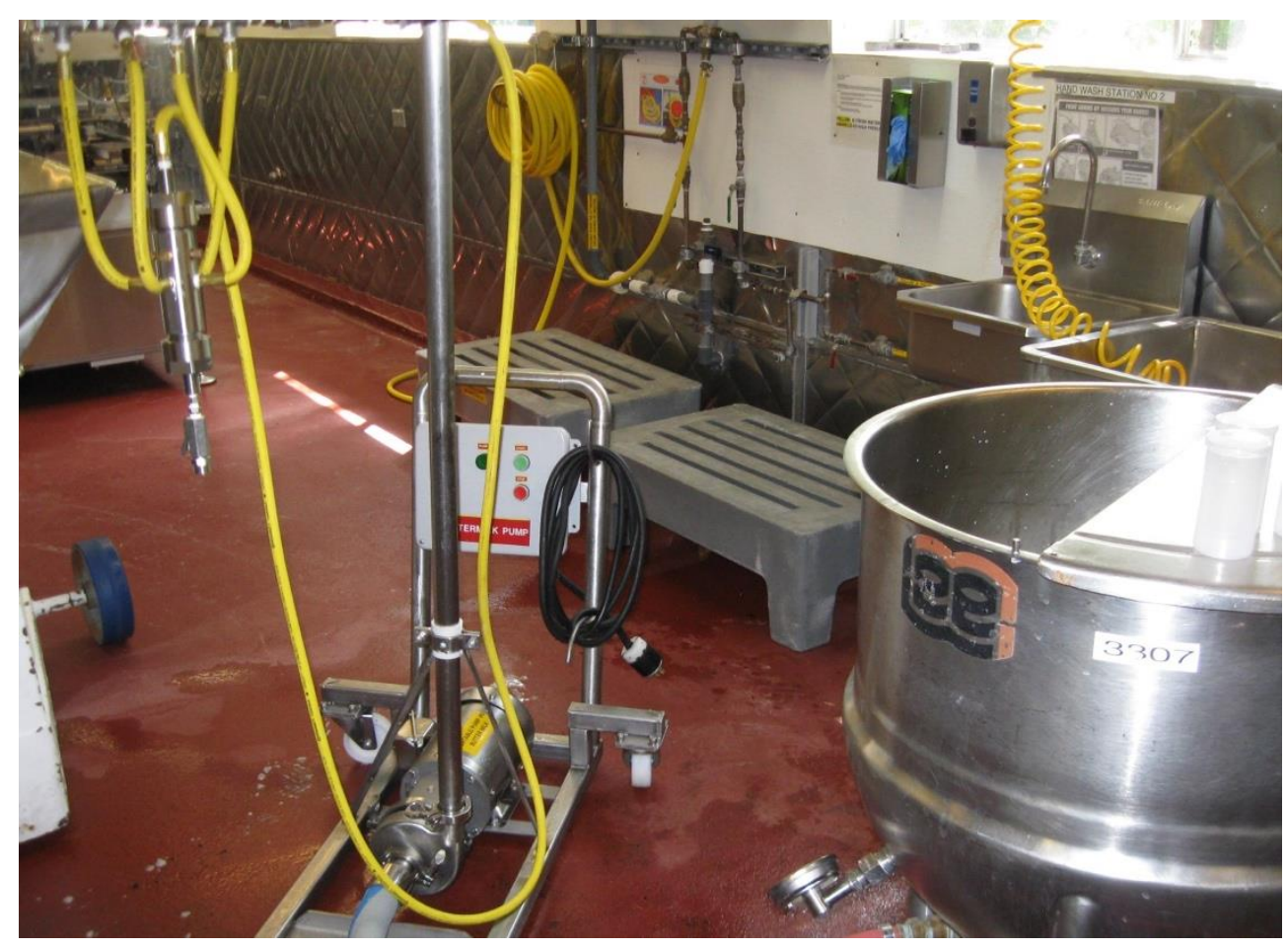

Figure 7. The figure shows an experimental composite mobile system for homogenizing milk at low pressure; Comprehensive tests of this system make it possible to take into account, when switching to stationary systems, all the necessary parameters including, first of all, longevity and strength of such equipment, taking into account the potential high level of wear of the working surfaces of the system and safety requirements during its maintenance and operation

There is another subjective longevity factor to be taken into account. The stereotypes of longevity formed for different types of products, determine many commercial factors, including the number of products required and, therefore sold, and their actual price. Imagine that you have found a technical solution to increase the product longevity and in this case this factor reduces the amount of the required product, while maintaining the existing level of price that the consumer is willing to pay for this product.

This leads to a decrease in sales volumes of companies making the product and puts these companies before a choice - to accept innovation or do everything to block the implementation and introduction of innovation. As practice shows, these companies choose the second option 
and block innovation, and in this process, the only loser is the inventor who invented something rejected by the market.

\section{A new look at the new product reliability}

The issue of the new product reliability and the new criteria for evaluating and calculating reliability also undergo fundamental changes. First of all, it is the connection of reliability and warranty obligations of the new product manufacturer before the consumer. Very often the cost of performing warranty obligations is comparable to the cost of the product itself.

That is, reliability is a factor that, being one of the achieved objectives of the invention, can determine (naturally, in combination with other technical and operational factors achieved as a result of the invention implementation) the level of commercial success.

In this case, the subjective factor of time also plays an important role, and more than the required reliability over time, can become a negative factor and play a cruel joke with the inventor in a situation when the created ultra-reliable product turns out to be commercially unprofitable for the manufacturer.

\section{New features in efficiency of system search and analysis of previous technical solutions for novelty}

It is clear that new information technologies open up new opportunities in the system search for similar solutions when elaborating a developed technical solution.

Imagine that, with the preliminary design of the structure of the composition, we came to the need to combine and integrate several classical solutions and new, say, digital technologies connecting them into the composition, so to speak, at the same level of horizontal integration, and after that, we also came to the need to go to the next level of integration with the inclusion in the composition of algorithms, software products and interfaces for communication with the previous level of integration.

How to search, in what directions, and do it most effectively and identify existing analogues of the composition being created. 
In this case, it seems to me that it is most probable to start a search, after forming the content of the composition being created, to begin a system search, after decomposition and identifying independent technical solutions included in the composition.

After this stage of the search, it is better to choose one basic technical solution from these technical solutions and upon searching through it, start to attach other technical solutions to the basic solution, that are included in the composition, and conduct a consistent search through the basic technical solution with each technical solution attached and so on until achieving full composition.

New features for assessing the usefulness and possibility (as well as expediency) of modifying and modernizing known technical solutions

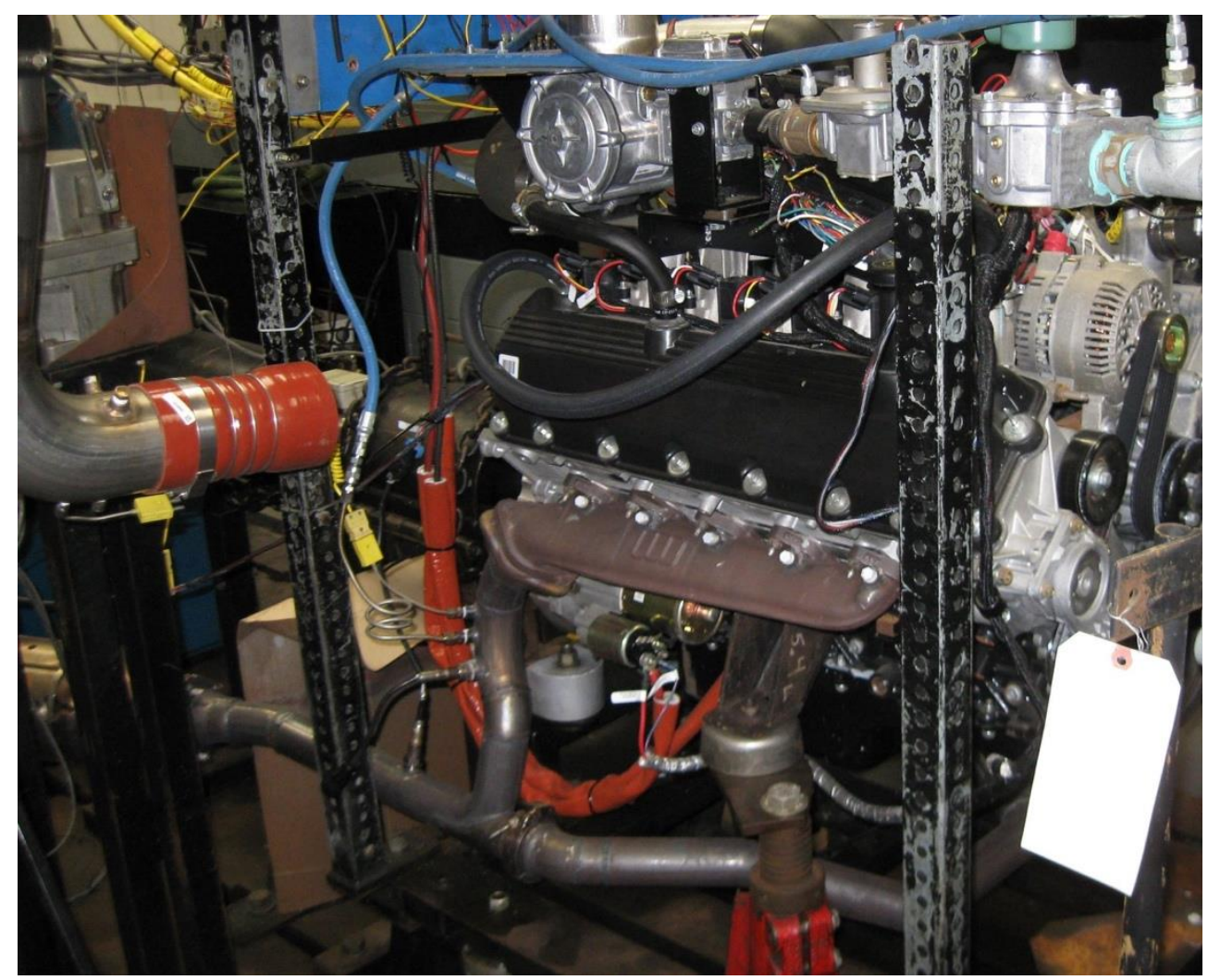

Figure 8. The figure shows the working elements of the engine of a backup electric generator installed on a dynamic stand, being modernized to change the type of reserve fuel from gasoline to natural gas; The installation on the stand was planned in such a way that, 


\section{first of all, to make sure that there are all conditions and technical grounds for modernization.}

Very often the new is well forgotten old...

Therefore, when setting a task and deciding on the start of an innovation process for synthesizing a new product, it is advisable to check whether some functional elements of the composition that need to be invented have been previously invented.

If such or an equivalent solution is found, then the replacement of materials, the use of new components and the introduction of a digital monitoring and control system into a future composition will make it possible to create a new technological composition that has the potential to integrate into a higher level composition.

\section{Impossibility of successful commercialization without forming the principles of} composite technical and integrated technological solutions, and the composite structure of a new technical solution with all the differences and properties

As practice shows, the possibility of selling or licensing autonomous technical solutions, in the event that they are not previously tied to systems or solutions of a higher technological and qualitative level, is reduced to zero.

To ensure the guaranteed commercial potential of such integrated solutions, the development of design, engineering and technological documentation requires specially planned stages of end-to-end computer modeling and simulation of internal vertical and horizontal integrative connections of the new development, and the program of such modeling should be one of the basic parts of Initial Technical Requirements for the product and must be taken into account in the Terms of Reference for the new product.

If the field of use of the new product is regulated and limited to the requirements of the relevant standards, including international ones, it is necessary to take these requirements into account in the process of preparing and agreeing on the Initial Technical Requirements and the Terms of Reference for development.

As a rule, composite technical solutions are more flexible in all respects, which allows them to be tied to the requirements and restrictions of standards at all levels and to avoid adjustments and problems at the stages of commercialization. 


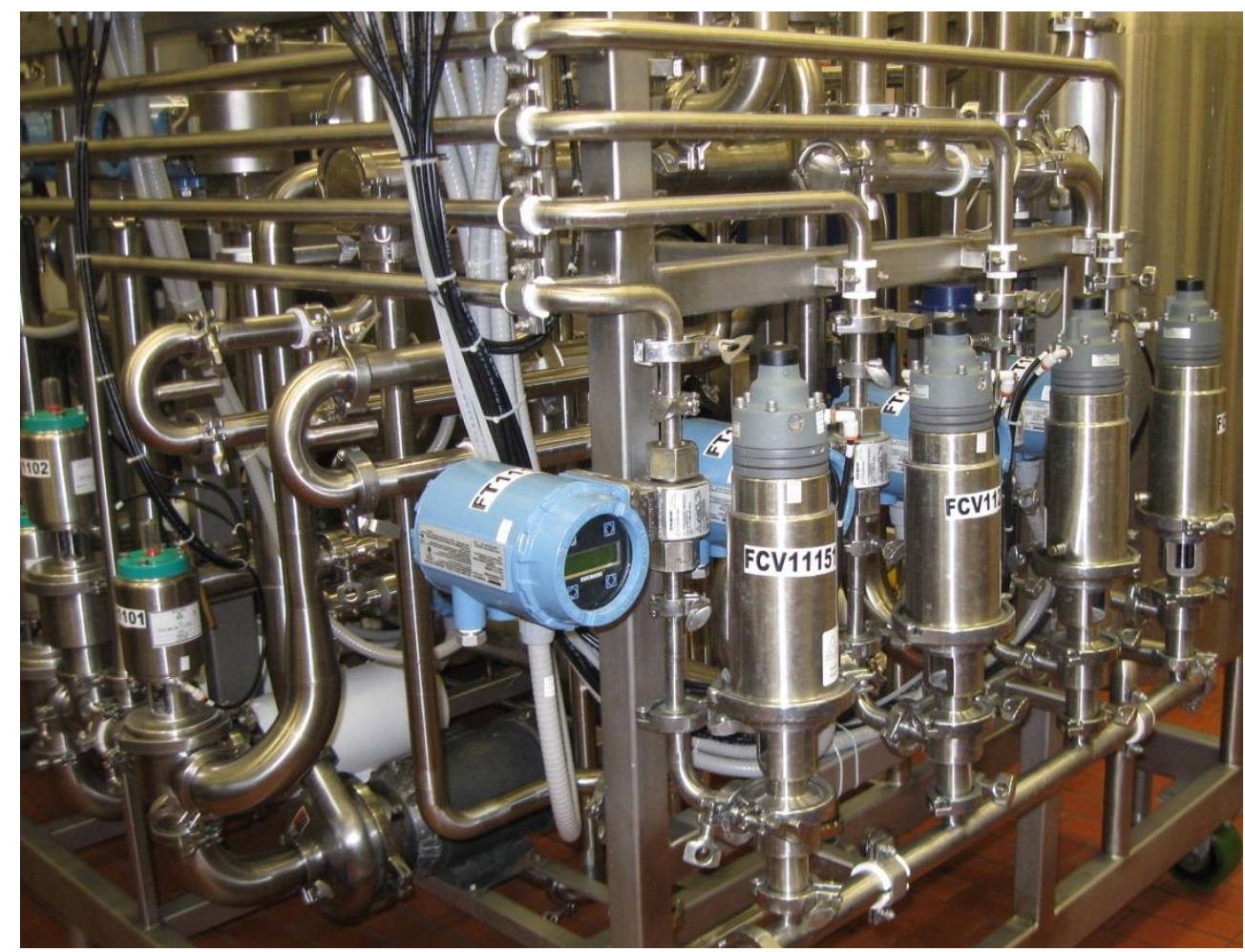

Figure 9. The figure shows the structure of a flexible production and technological module in the production process of dairy products, where autonomous components are applied, each being a composite technical solution and in turn contains a structure consisting of a complex of integrative inventions that have a coherent integration with adjacent elements of the entire technological system both in the horizontal version, and in the vertical version, but at the same time each such component in the system hierarchy has a certain degree of autonomy and delegates to each adjacent component a part of its control functions within the framework of the end-to-end technical characteristics of the system and under the exchange protocols between the adjacent components in the entire kinematic scheme of the entire system of flexible automatic modules altogether comprising the base for all production cycle.

Inventions of a composite nature, having at least a principal schematic solution for integration into technological and structural systems of a higher functional level, are implemented more confidently and in a shorter time, because investors, buyers or consumers 
of the license have the methodology and technique of this integration in the description and in the formula of this integrative and composite technical solution.

\section{Proposed techniques and methods of forming a composite style in creation of new}

\section{innovative solutions}

Thus, the composite structure of a technical solution is the engineering and design style of developing new technical solutions for incorporating them into existing process schemes and configurations.

Since the technique of such incorporation can often be unique and have a significant novelty, the description and formula of the invention of a composite nature, a multi-level architecture for building causal relationships between the components of the composition and distinctive features integrated into the design and technological relations of the composition, determine the commercial success of these innovations to a large extent.

Techniques and methods of transition from the created composite foundation of a new technical solution to the basic foundation of an integrative invention

The design and technological composition in many cases requires additional, often fundamentally new relations between the components and elements of the composition, in other words, often having a design and technological composition clearly expressed in terms of properties and content, in order to turn it into a finished innovative product or item, fit is necessary to find versions of the composition integration into this final and repeatedly and at many levels systemically integrated product. 


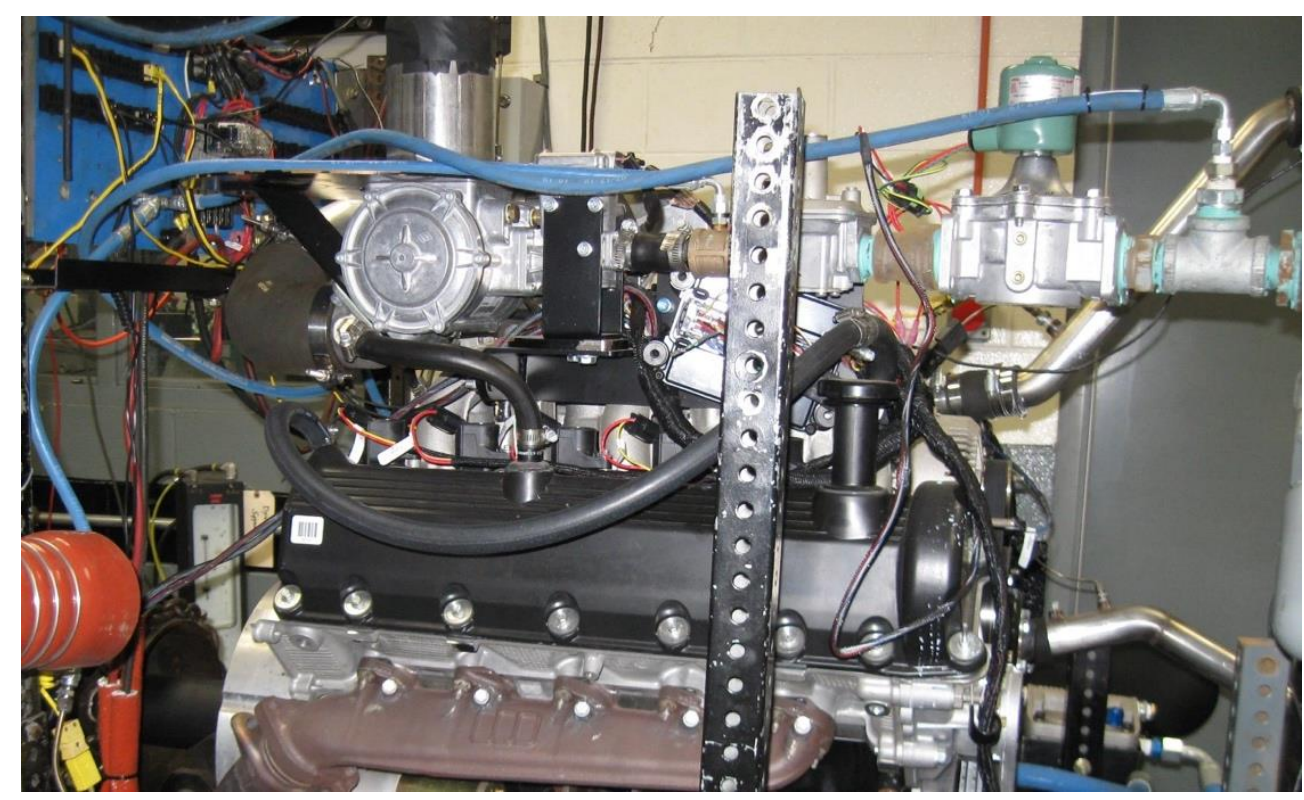

Figure 10. The figure shows a fragment of a gasoline generator prepared for switching the engine fuel system from gasoline to natural gas, and natural gas must be supplied to the combustion chambers of the engine in the form of a vortex tube formed in the course of the natural gas flow along the fuel line where the integrative inventions of the previous stage off engine modification and optimization, disk vortex generators, are mounted; In the same principal configuration of the addition to the engine fuel system, a natural gas flow can be mixed with compressed air within the same vortex tube, in order to reduce air consumption for stoichiometric mixing and at the same time reduce potential carbon monoxide exhaust and also reduce overall consumption of natural gas while retaining the previous thermal and dynamic performance of the engine and to reduce hazardous exhaust concentration 


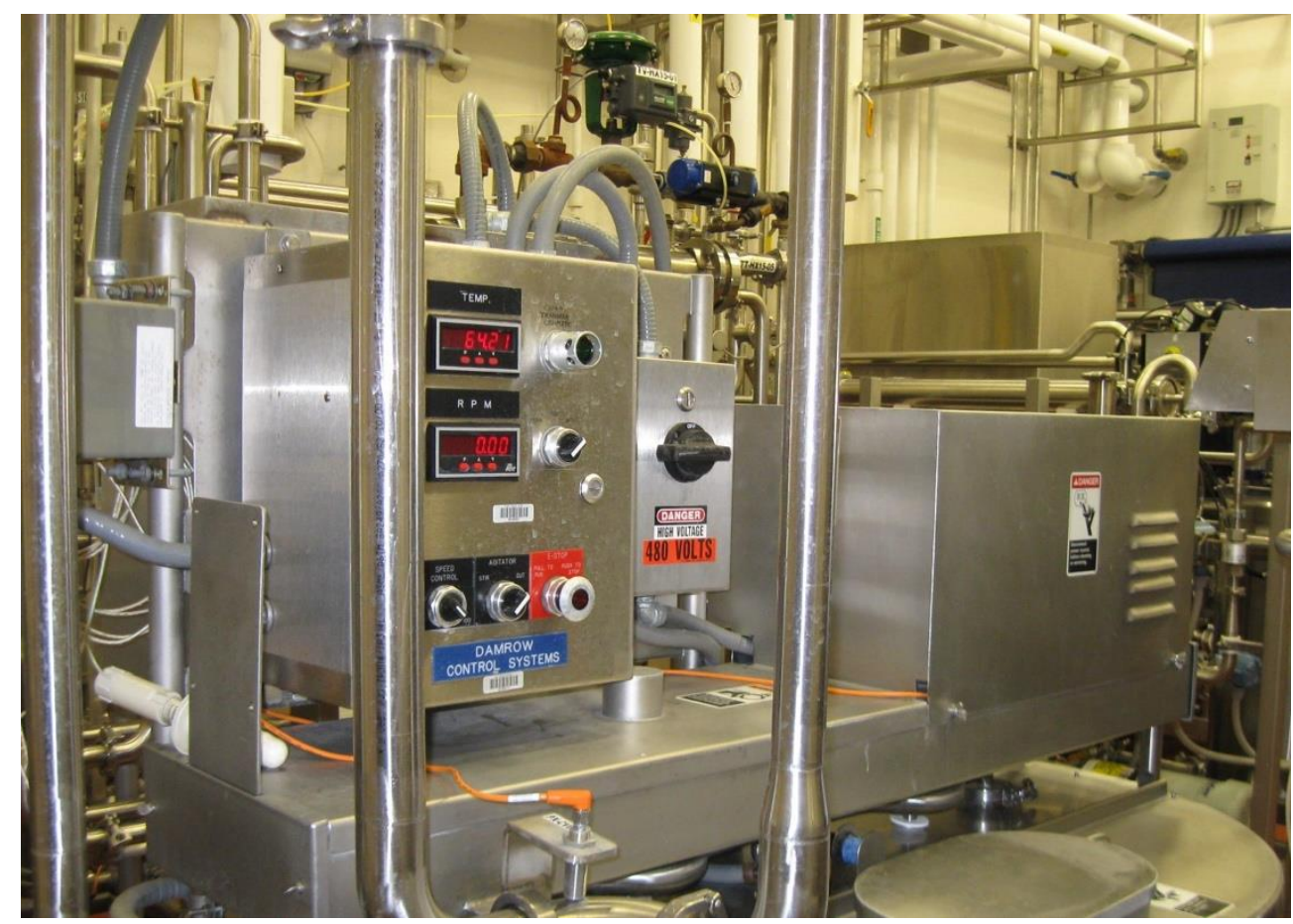

Figure 11. The figure shows a fragment of the system of composite integrative inventions in general forming flexible automated dairy production in a closed automatic cycle and with full control of all operations at the level of process parameters, detailed to the parameters of each technological operation and successively to each technological transition and measuring local stage.

Versions of integration can be very different; it is only important that the end result of the integration was a leap of the effect or a leap of the quality having no precedent earlier.

The effect of restrictions on the number of paragraphs of the invention formula on the possibility of reliable protection of composite technical solutions.

Restricting the number of invention formula paragraphs in principle makes it difficult to reliably protect an invented item, but a correctly found principle of a composite solution can, on the contrary, increase the degree and level of protection.

The ideal case is a system of causal relationships to get the declared effect only in the proposed system of composite relationships with clearly defined conditions and signs that 
determine the content and independent functions of each of the elements of the resulting composition.

Due to limited space and form, it makes sense to select only those signs and relationships in the composite solution that do not affect the well-known independent features and functions of each of the elements and components of the composition, but arose as a result of the composition formation from the fields of functional influence of the composition components on each other.

It can be said that in the correctly selected composition components, when they are subordinate within the framework of the composition to the conditions and properties of the newly created technological system, a new integrated system of signs, relationships, feedbacks and functions, possible only within the framework of this composition and, moreover, having a tendency to the development and improvement of intra-compositional relations, arises.

Following these principles, within the framework of a limited number of formula paragraphs, allows focusing only on the main distinguishing features of the composition, while ensuring the maximum level of protection of the composition and the maximum level of understandability of the invention essence.

\section{The proposed structure of the independent paragraph of the invention formula} built on the basis of a composite technical solution

As I determined as a result of my first experiments and as recommended by specialists, the independent paragraph of the invention formula, if the invention is a composition, should have at least three main parts.

The first part carries formulates commercial essence of a compositional invention and should disclose the meaning and necessity of compositional integration for:

- accurate formulation of the composition task

- limiting the degree of functional relationships in the composition and disclosing the degree of necessity of each composition component for its formation and normal and effective functioning

- formulation of the composition name

The second restricting part includes all the basic information about the invention as such and includes the characteristics of all basic technical solutions inherent to the composition 
components and at the stage of drafting the invention formula not qualified for the presence of essential novelty elements in all design and technological aspects and relations of the composition.

The third distinctive part includes information about components, their relationships, materials, integrative elements and associated software products and their basic algorithms, each of them, independently or in any combination, creates elements of significant novelty for composite, multiple (and integrated at many levels) technical solutions of any complexity and structure.

The proposed structure of the dependent paragraph of the invention formula built on the basis of a composite technical solution

Restricting the number of paragraphs of the invention formula imposes a special mission in the overall strategy of formulating and protecting the novelty of an invention in each paragraph of the formula.

Based on it, the second restricting part of such paragraph of the invention formula shall include all the basic local and specific information about the invention as such and includes local and specific characteristics of all basic technical solutions inherent to the composition components and at the stage of drafting the invention formula not qualified for the presence of essential novelty elements in all design and technological aspects and relations of the composition.

The third distinctive part includes local information specifically focused on technical details and elements, about components, their relationships, materials, integrative elements and associated software products and their basic algorithms, each of them, locally, independently or in any combination, creates locally specific elements of significant novelty for composite, multiple (and at many technological and constructive levels) complex integrated technical solution.

The proposed methodology for designing prototypes of composite technical solutions to test and verify the correctness of each innovative element of the composition

Designing of a prototype or a pilot sample of an innovative product-composition is most convenient when using the design program and its engineering analytical applications. I prefer 
the Solid Works software product, since this tool can build a valid model of a composite technical solution and conduct a control simulation of its operating cycle, without resorting to expensive design and making prototypes.

\section{Analysis of found analogs and prototypes of a new composite technical solution}

If, as a result of the search, homogeneous, basic technical solutions are found in the first approximation, being analogs or prototypes of the conceived composite technical solution, they should be tested in various versions and combinations of integration with additional elements and components of the considered composition.

If information about these prototypes or analogs is available in the respective storage media (including in digital format), then it is advisable to use Solid Works tools to build models

of the found technical solutions and to conduct a digital simulation of the working cycles of these models to compare them with similar working cycles of the proposed invention - a composite technical solution.

\section{Examples of composite technical solutions perceived by the market}

Examples of composite technical solutions today are products of computer technology, communications equipment, tablet computers and many other products of mass and non-mass demand, well-known to everyone.

Uncertainty in wording of patent applications as the most complex technical solutions underlying these products has led and leads to numerous patent disputes and wars.

More extensive use of composite technical solutions and their integrated extensions and interpretations will reduce the intensity of passions and may help in promoting innovative products to the market.

Of the most well-known compositional technical solutions, the most relevant to all of the above, today are widely known iPhone smartphones produced by Apple.

Due to its complete composite three-dimensional constructional structure and full integration into all possible fragments of the existing external information infrastructure, this stunning multi-functional device shows unprecedented commercial success in world markets. 


\section{Compositional technical solutions as a set of useful functions of a technical system}

(TS)

Main useful function. For a set of useful functions of TS, we can always find a more general useful function that directly reflects the TS purpose, the purpose of its existence and activity (and coinciding with them).

This general TS function is called the main useful MUF of the whole TS, in contrast to the elementary useful functions (hereinafter referred to as simply useful, UF), which together ensure the implementation of MUF. The relationship between MUF and UF is the same as between the system and its subsystems. MUF refers to the system as a whole, and UF to its subsystems.

Positive effect. Any change in TS that increases the capabilities of this TS in meeting the needs of super-systems (and society as well) is an improvement of the system.

The improvement in TS is manifested in the following changes in the system at the level of external functioning:

- the quantitative growth of useful "capabilities" of TS - transformation of unuseful "capabilities" into useful ones;

- elimination of harmful "capabilities" until they turn into useful ones;

- increasing the ratio of the useful output to the input, i.e., increasing the TS efficiency.

Dialectical contradiction. The source of development of TS, as well as any object of the material world, is the law of unity and struggle of opposites - the universal law of development of nature, society, technology.

Opposites are the sides of an object that are in mutually exclusive relationships. In this case, the side of an object or phenomenon is understood as everything that is somehow inherent in the object or phenomenon, characterizes it and can be comprehended.

Opposites in TS are "input" and "output", useful functions, costs and "capabilities".

The interaction of opposites, when they both mutually presume and at the same time deny, exclude each other, is a dialectical contradiction.

Technical contradiction (TC) is a dialectical contradiction, which manifests itself in the technical system as a deterioration of one side of TS at the level of external functioning (from the standpoint of super-system needs) while improving the other side of TS. 
In other words, TC can be defined as the dialectical unity of interdependent positive and undesirable effects in TS. TC is always associated with some component of TS (element, group of elements, or interaction of elements), which is commonly called the nodal component (NC).

This TS component is immediately related to two sides of TS, and a quantitative change in some parameter (or state) of this component leads to an improvement in one and a deterioration in the other side of TS.

Therefore, more precisely, TC should be defined as the dialectical unity of positive and undesirable effects interdependent on quantitative or qualitative changes in the nodal component of TS.

The inventive problem arises in case of an exacerbation of TC inherent in TS. At the same time, improvement of some "capabilities" of aTS due to a quantitative change in some parameters becomes impossible due to a significant deterioration in other "capabilities."

Attempts to preserve TS due to a compromise between opposing sides in this case are not successful. The resolution of TC is possible in case of a transition of TS to a new qualitative state - a dialectical jump. This is an invention.

When considered from a more general perspective, the problem of resolving the contradiction between public need and the possibility of satisfying it can be reduced to one of two tasks:

a) search for a material form based on the laws of nature and allowing to perform a function corresponding to a specific public need - an information task (search for a new system);

b) resolution of the internal dialectical contradiction in a technical system that satisfies a certain public need - a problem-contradiction.

These two types of tasks are related to each other and in practice of technical creativity they go one into the other.

Physical contradiction. The technical contradiction in its form appears in TS at the level of its external functioning. At the level of internal functioning, no mutually exclusive relations between the parties to the system is observed: from a physical point of view, TS is in some one state determined by the laws of nature.

But if we set the task of eliminating TC within this TS, asserting positive and negating undesirable effects, then mutually exclusive relations will manifest at the level of internal 
functioning, in the form of incompatible requirements for the parameter (state) of the nodal component of TS, more precisely, to the physical state of NC.

Such contradictions are called physical (FC). FC manifests itself in formulation of the task of eliminating TC, in other words, FC is a form of expression of the problem of eliminating TC in the framework of this TS. FC resolution is to establish new forms of organization and movement of matter in TS, when both incompatible requirements for the NC state are met, to establish such a "form of movement where this contradiction is both realized and permitted."

\section{Introduction to the theory of invention problem solving (TIPS)modified based on}

the recent conditions for the development of unified innovative systems and complexes

1. Creative activity is usually determined through the result. As an example, one of the most common definitions can be given: "Creativity is a human activity that creates qualitatively new material and spiritual values."

If we try to formulate the definition of creativity as a process, we will see that creative activity is the process of finding a solution.

In essence, all human activity can be divided into two large areas: area of routine operations and area of problem solving.

Representation of creativity in the form of a problem-solving process makes the following conclusion self-evident: in order to scientifically organize creative activity, one must first of all put the problem-solving process on a scientific basis. In other words, TIPS is required.

2. Problem solving is of two types: strict and nonstrict. Strict solutions are based on complete reliability, accurate information and, as a rule, are quite unambiguous. Solutions obtained on the basis of incomplete, inaccurate information, under conditions of uncertainty, are called nonstrict.

Accordingly, methods for obtaining solutions are divided into strict and heuristic methods. When solving problems put forward at the present level of social development, these methods complement each other.

As science develops, many heuristic methods of solution are formalized and transferred to the strict class according to the following scheme: accumulation and systematization of 
knowledge - development of "intuition", intuition - formalization, development of the theory - the algorithm.

3. The existing problem-solving apparatus is adapted to the search for rigorous, quantitative solutions. It includes such sciences as system analysis, solution search theory and decision theory. The basic idea of system analysis is the following: "The solution to any problem is the process of creating a new system."

System analysis is based on systems engineering (construction of large technical systems) and organizational systems engineering (systems engineering of organizations).

Decision theory considers methods for finding the best ways to achieve goals.

It includes such disciplines as operations research (the use of mathematical, quantitative methods to substantiate solutions in all areas of targeted human activity), a linear programming method (choosing the optimal solution from a large number of possible).

The solution search theory considers the process of searching for a solution in conditions of uncertainty in terms of information.

4. TIPS deals with the search for complex composite heuristic solutions. Its main features include the following:

a) the theory should provide a significant increase in the probability of getting the right solutions;

b) the theory should be engaged in search for solutions at a qualitative level;

c) the theory should take into account the characteristics of the object and the subject of creativity.

TIPS meets all the above requirements. In addition, it is based on two main provisions:

1. A new, truly creative solution in engineering corresponds to the next stage in the development of the object which the solution relates to.

2. The patterns of the technology object development process are knowable and can be used to search for new technical solutions.

The feature factor is what is inherent only in a given theory, is most characteristic of it, and distinguishes this theory from similar fields of knowledge. For TIPS, the feature factors are:

- the use of the identified patterns of construction and development of technical systems; 
- availability of optimal logic for identifying the problem and finding new technical solutions.

\section{References}

1. US patent application No20130132320, 23.05.2013. INNOVATION EXPERT SYSTEM, IES, AND ITS PTR DATA STRUCTURE, PTR-DS / Schindler; Sigram.

2. US patent application No20130161508, 27.06.2013. METHOD OF MASSSPECTROMETRY AND A DEVICE FOR ITS REALIZATION / Sapargaliyev; Yerbol Aldanovich; et al.

3. US patent application No20130223721, 29.08.2013. SYSTEMS AND METHODS FOR DEVELOPING AND VERIFYING IMAGE PROCESSING STANDARDS FOR MOBLE DEPOSIT / Nepomniachtchi; Grigori; et al.

4. US patent application No20140052649, 20.02.2014. Data Management System for Generating a Report Document by Linking Technical Data to Intellectual Property Rights Data / Prat; Jordi; et al.

5. US patent application No20140321560, 30.10.2014. METHOD AND TECHNICAL EQUIPMENT FOR VIDEO ENCODING AND DECODING / Ugur; Kemal; et al.

6. US patent application No20150248321, 03.09.2015. CYCLICAL METHOD AND A DEVICE FOR LOCALIZING UNCONTROLLABLE MULTIPLE FAILURES IN ENGINEERING SYSTEMS IN OPERATION / BUKOV; Valentin Nikolaevich; et al.

7. US patent application No20140359566, 04.10.2014. RESOLUTION OF TEXTUAL CODE IN A GRAPHICAL HIERARCHICAL MODEL OF A TECHNICAL COMPUTING ENVIRONMENT / AVADHANULA; Srinath; et al.

8. US patent application No20150317528, 05.11.2015. SYSTEMS AND METHODS FOR DEVELOPING AND VERIFYING IMAGE PROCESSING STANDARDS FOR MOBILE DEPOSIT / Nepomniachtchi; Grigori; et al.

9. US patent application No20160059412, 03.03.2016. ROBOTIC MANIPULATION METHODS AND SYSTEMS FOR EXECUTING A DOMAIN-SPECIFIC APPLICATION IN AN INSTRUMENTED ENVIRONMENT WITH ELECTRONIC MINIMANIPULATION LIBRARIES / OLEYNIK; Mark. 
10. US patent application No20160266576, 15.09.2016. CABLE PROCESSING MACHINE MONITORING WITH IMPROVED PRECISION MECHANISM FOR CABLE PROCESSING / Ayabakan; Mustafa; et al.

11. US patent application No20170280351, 28.09.2017. METHODS, NON-TRANSITORY COMPUTER READABLE MEDIA, AND SYSTEMS FOR IMPROVED COMMUNICATION MANAGEMENT OF A PLURALITY OF WIRELESS NODES IN A WIRELESS NODE NETWORK / Skaaksrud; Ole-Petter.

12. US patent application No20180129784, 10.05.2018. SYSTEMS AND METHODS FOR TECHNICAL SUPPORT OF CONTINUOUS ANALYTE MONITORING AND SENSOR SYSTEMS / Pal; Andrew Attila; et al.

13. US patent application No20180248777, 30.08.2018. CONTEXT AWARE STREAMING OF SERVER MONITORING DATA / Chakra; Al; et al. 